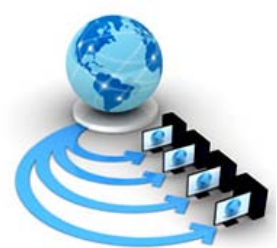

Volume 8, No. 7, July - August 2017

RESEARCH PAPER

\title{
OPTIMIZATION AND IMPLEMENTATION OF WIRELESS SENSOR NETWORK PERFORMANCEUSING ANT BEE COLONY
}

\author{
Anu Kadian \\ Assistant Professor. \\ UIET Meharshi Dayanand University, \\ RohtakMDU Rohtak,Rohtak, Haryana
}

\begin{abstract}
Routing in wireless protocols is a tedious task. A wireless node is never stationary. It is moving from one location to other which makes traditional routing algorithms like OSPF, RIP inefficient in wireless routing. Aim is to produce a routing algorithm which works efficiently with constantly changing locations of the mobile node by implementing traditional biological background based ant bee colony technique.Traditional biological background based ant bee colony is used.A efficient ant bee colony based routing algorithm for Mobile Ad hoc Networks is obtained. Shortcomings in implementation of above algorithm are also discussed.
\end{abstract}

Keywords :Bee colony,Wirelwss sensor network, optimization

\section{INTRODUCTION}

Nature-Inspired Algorithms are the most studied research area by various computational researchers and its inspired by the various variety of biological and natural processes. The popularity of the Nature-Inspired Algorithms is mainly initiated by the ability of biological systems to efficiently adjust to frequently changeable environment. Evolutionary Computation, Neural Networks, Bee Colony, Ant Colony Optimization, Particle Swarm Optimization, Artificial Immune Systems, and Genetic Algorithm, are the algorithms and concepts that were inspired by nature. Swarm intelligence, as a scientific discipline including research fields such as swarm optimization or distributed control in collective robotics, was born from biological insights about the incredible abilities of social insects to solve their everyday-life problems. The roots of swarm intelligence are deeply embedded in the biological study of self-organized behaviours in social insects. SI is the name given to the mechanism or system where in a group of workers/particles/agents work in collaboration with each other to find optimal solution for the problem in hand. Various examples of SI groups are bird flocks, bee colony, ant colony, particle swarm, cockroaches, fishes, etc. $[1,2,3,4]$ SI systems have strong features like group defines as is in bee colony, labour division as in ant colony, synchronization as in flying birds' flock, collective clustering and sorting, cooperative working etc. In SI the agents form or build a local solution, on the basis of factors/conditions in hand. Based on the local solution, the global solution is developed which then gradually optimizes or channelizes to an optimal solution. So, SI is a bottom-up type of problem solving technique. [1] In this paper a survey on the areas of applicability of bee colony system have been presented.

II. BEE COLONY The BCO is inspired by the bee's behaviour in nature. The basic idea behind the BCO is to create multi agent system (colony of artificial bee) capable to solve different combinatorial optimization problem. The artificial bee colony

behaves partially alike, and partially differently from bee colonies in nature. The bee system is a standard example of organized team work, well coordinated interaction, coordination, labour division, simultaneous task performance, Specialized individuals, and well-knit communication. [2,5,6,7] In a typical bee colony there are different types of bees. There is a queen bee, many male drone bees and thousands of worker bees. [2] Types of bees: [2] 1. The Queen's responsibility is of laying eggs so that new colonies can be formed. 2. The Drones are males of the hive and are responsible to mate with the Queen. This is their sole role in the hive. They are discarded from the colony during their down fall. 3. The worker bees are the females of the hive. They are the main building blocks of the hive. They build the honey bee comb, clean it, maintain it, guard it, feed the queen and drones. Apart these side responsibilities the main job of a worker bee is to search and collect rich food. There are two types of worker bees namely scout bees and forager bees. Both of them are collectively responsible for the collection of food but they play different roles. What does Scout do? $[8,9,10] 1$. The Scout bees fly around and search for food sources available randomly. 2. They return back to the hive after they exhaust their energy and distance limits. 3. Upon returning to the hive they share their exploration experience and a lot of important information with the forager bees. 4 . The scouts tell the foragers about the location of rich food sources which comprises of the direction (angle) of the food source from the hive w.r.t. sun and distance from hive. This is done using a dance called "waggle dance" which is in the figure of digit "8". It also indicates the quality of food. What does Forager do? The forager bees closely observe the scout bee in order to learn the directions and information given by scout. It then goes to collect food. Artificial Bee Colony (ABC) was introduced by Karaboga in 2005. It was developed to solve real parameter optimization problem. In ABC the BCO's 
foraging behaviour is simulated. The ABC differs from a real $\mathrm{BCO}$ since in $\mathrm{ABC}$ we use only scouts and foragers in equal proportion as initial population. The main steps of ABC are: 1. Initialization of food sources. 2. Scouts perform exploration of available food sources randomly until stopping criteria is met. 3. Each Forager exploits the respective scout's food sources until stopping criteria is met. 4. Forager chooses the best food source as per quality

\section{WIRELESS SENSOR NETWORKS}

One of the main design aims of WSNs is to transfer data communication while trying to extend the lifetime of the network and avoid connectivity degradation by employing aggressive energy management techniques.[11] Due to the limited range of communication, ensuring the direct connection between a sensor and the base station may make the nodes transmit their messages with such a high power that their resources could be quickly depleted. Thus, the collaboration of nodes ensures the communication between distant nodes and base station. In this method, intermediate nodes transmit messages so that a path with multiple links or hops to the base station is established. Collaborative work between sensors requires an intelligent organization to transmit information from the sensing field to the base station in order to save energy re-sources of the network. Because of the insignificant computational capability and the lack of energy sources, the Flooding algorithms are not a proper solution for routing of WSN application. The flooding algorithms broadcast the data to all overlapped nodes to the extent that cause an implosion and some nodes redundantly receive multiple copies of the same message. Gossiping algorithm comes with a better performance, avoiding implosion as the sensor and sending the message to a selected neighbor instead of informing all of its neighbors. However, it is still not the proper solution.

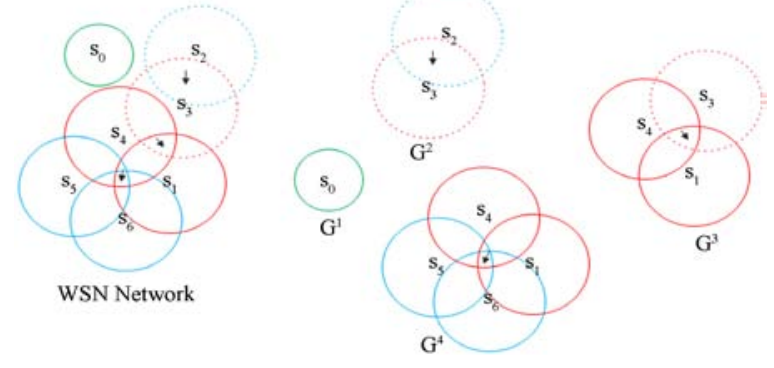

Figure 6. Partition the WSN nodes into routing group.

\section{LITERTURE REVIEW}

Guikai Liu et. Al., in [11] Multi-hop wireless ad hoc network, also called multi-hopwireless self-organizing network, does not rely on a fixedinfrastructure and the network structure changes dynamicallydue to member mobility. Wireless ad hoc networksare very attractive for tactical communication in militaryand also expected to play an important role in manyfields without the presence or use of a fixed infrastructuresuch as disaster search-and-rescue operations, data acquisitionin remote areas, conference and convention centersetc.
Minghui Li et. Al., in [12] Estimation of the incident signals' directions, or angle-of- arrival (AOA) estimation, is a fundamental problem in numerous applications such as radar, sonar, radio astronomy, and mobile communications. AOA measurements can locate mobile units, and thus support and enhance location-aided routing, dynamic network planning and management, and different types of location-based services and applications, furthermore, it can improve communication efficiency and network capacity when integrated with adaptive array technology[13].

Ka Lun Lam et. Al., in [14]The ZigBee protocol was originally defined for non-mo- bile applications and has gained increasingly importance recently. Pilot works on ZigBee did not discuss the de-sign of large scale system which demands mobility. There are increasingly more applications which demand mobility within a large ZigBee fixed network, say for patient monitoring application in hospitals and aged- person caring centers. Up to present, there has been no study on enabling mobility in a fixed ZigBee network. It will soon be explained that such mobility enabling is different from the traditional ZigBee ad hoc networks in terms of topology and addressing requirement. Aging tends to be a serious worldwide problem in coming 20 years. Health care for aged persons will be a very alarming issue. It is insightful to understand the pragmatic application of ZigBee by studying the de mands of future health care for inpatients and outpatients within hospitals.

Wei Huang et. Al. in [15] proposed that flood disaster is one of the natural calamities which have the greatest effect, the highest frequency and the most severe loss . Because of the high dimension and complexity of the flood disaster system, there are no uniform flood disaster assessment indices and technical methods, and flood disaster assessment is still one of the difficult and hot spots in flood disaster research. Flood disaster assessment is to search the non-linear relationship between flood disaster assessment indices and flood disaster grads on the basis of having obtained some disaster assessment indices, so that it can assess the loss degree caused by the flood disaster, which is a pattern recognition problem essentially. In order to estimate the flood disaster objectively and accurately, multi-factor assessment model need to be built. In terms of the flood disaster classification assessment, several methods, such as matter-element analysis method, fuzzy synthetic evaluation method, gray cluster, artificial neural network, and immune genetic algorithm, have been proposed . However, the results estimated by those models are discrete disaster degrees, and the disaster resolution is low.

Mark Hartong et. Al., in [16]proposed the primary objective of inter-domain rail operation is tominimize rail traffic delay at interchange points whilemaintaining safe operating conditions. Delays add to arailroads cost of business and can have a significant impacton the US economy. Techniques used to minimizedelays are categorized as tactical (i.e. addresses localscheduling decisions) and strategic (i.e. addresses globalscheduling decisions over regions).

Zhizhong Jie in [17]proposed Vehicular Ad Hoc Network (VANET) is becoming an active research area in recent years. VANET is a multi- hop mobile network designed to offer a wide range of road applications such as safety warning, congestion avoidance and mobile infotainment. VANET has particularly important applications in sparse 
and rural areas because of the lack of fixed communication infrastructure. That is the reason why routing algorithms appropriate for these circumstances and the design of such a routing protocol is challenging

Sathya P. Duraisamy et. Al., in [18] proposed In many image processing applications, the gray levels of pixels belonging to an object are substantially different from those belonging to the background. As such, thresholding techniques can be used to extract the objects from their background. Indeed, thresholding is a major operation in many image processing applications such as document processing, image compression, particle counting, cell motion estimation and object recognition. The effect of many image processing applications strongly depends on the effect of image thresholding. Thresholding techniques provide an efficient way, in terms of both the implementation simplicity and the processing time to perform image segmentation.

Fatemeh Ayatollahi et. Al. in[19]proposedMedical image registration plays an increasingly important role in many clinical applications, including the detection and diagnosis of diseases, planning of therapy, guidance of interventions, and the follow-up and monitoring of patients. Image registration is the process of overlaying two or more images taken from same scene at different times, from different viewpoints or by the variety of sensors.

Olatunde Abiona et. Al. in [20] proposed Wireless networks has been experiencing an explosive growth similar to the Internet, this is due largely to the attractive flexibility enjoyed by both users and service provider. Some of the benefits are: network coverage without the cost of deploying and maintaining wires, mobility support and roaming which grant the users "anytime", anywhere access to network. While the emergence of these new technologies can enable truly ubiquitous Internet access, it also raises issues with the dependability of the Internet service delivered to users. Basically Wireless Local Area Network (WLAN) can operate in two modes, the infrastructure based and the ad hoc networks. Many organizations are deploying the infrastructure based wireless network to provide connectivity to places difficult to reach by cabling, to complement the existing wired networks. Majing Su et. Al. in[21]proposed BitTorrent (BT) is one of the most popular P2P file- sharing systems and has a large amount of users. Despite BT is argued for copyright issues in recent years, the security and performance of BT systems still attract much attention. Researchers work hard to improve its performance. For example, extensions such as Distribute Hash Table (DHT) and Peer Exchange (PEX) have been applied in BT to ease the bottleneck of trackers. uTP is used to improve download speed.

Wenping Chang in [22] proposed Optimal operation is one of the important optimizationissues in a hydrothermal power system. Its objective is tominimize the operation cost of thermal units in a givenperiod of time while all constraints are satisfied. Sincethe cost of power generation is huge, the economic consequenceof operation scheduling is significant. A numberof methods have been proposed for solving thehydrothermal scheduling problem. Among these methods, DP wouldprovide a good framework for optimizing the decisions.

Magdi S. Mahmoud et. Al. in[23] proposed Dynamic system stability is a fundamental property of power systems that describes its ability to remain in a state of equilibrium under normal operating conditions and to regain an acceptable state of equilibrium in face of an external disturbance. It is generally observed that power system stability margins generally decrease, mainly due to: 1 )

\section{Problem formulation -}

1. Traditional routing algorithms performing poorly in wireless nodes due to constantly changing topology of mobile nodes.

2. Deploying a efficient wireless routing algorithm that caters for mobile topology characteristic of wireless nodes and providing a efficient routing algorithm using ant bee colony.

3. Comparing routing algorithms and observing performance enhancement in particle ant bee colonyalgorithm.

4. We can see a definite improvement in every aspect of derived output.

\section{Leach protocol -}

5. The LEACH routing protocol is developed by Dr. Wendi Rabnir Heinzelman in 2002. LEACH uses a periodic distributed clustering function to balance energy costs throughout the network. Time is divided into rounds, and every sensor has a certain chance of self-electing itself as a cluster head. LEACH is a clustering-based protocol that includes the following

6. features:

7. Randomized, adaptive, self-configuring cluster formation,

8. Localized control for data transfers,

9. Low-energy media access, and

10. Application-specific data processing, such as data aggregation.

11. Low Energy Adaptive Clustering Hierarchy (LEACH) is the first hierarchical cluster-based routing protocol for wireless sensor network which partitions the nodes into clusters, in each cluster a dedicated node with extra privileges called Cluster Head $(\mathrm{CH})$ is responsible for creating and manipulating a TDMA (Time division multiple access) schedule and sending aggregated data from nodes to the BS where these data is needed using CDMA (Code division multiple access). Remaining nodes are cluster members.

\section{Disadvantages of LEACH Protocol}

12. LEACH is able to perform local aggregation of data in each cluster to reduce the amount of data that transmitted to the base station. Although LEACH protocol acts in a good manner, it suffers from many drawbacks such like

13. - Cluster-Head selection is randomly, that does not take into account energy consumption.

14. - It can’t cover a large area.

15. - Cluster-Heads are not uniformly distributed; where Cluster-Heads can be located at the edges of the cluster.

16. - While the distributed algorithm for determining cluster-head nodes ensures that the expected number of clusters per round is $\mathrm{k}$, it does not guarantee that there are k clusters at each round. 


\section{Methodology used -}

1. Simulating the behavior of routing protocols using standard simulating tools

2. Studying and comparing various existing multicasting routing network protocols.

3. Deriving optimized ant bee colony routing protocol for wireless sensor network nodes/ MANET

4. Interpreting the performance graph of ant bee colonybased wireless routing algorithm

5. Discussing various problems/flaws usually encountered in implementing these algorithms.

\section{ANT BEE COLONY}

It is an algorithm analogous to movement of ants to cover the shortest path

1. Ant bee colony simulates the behaviors of ants to move in a straight line to cover the shortest distance to reach a destination.

2. An ant moving forward leaves its pheromone behind its existence.

3. Ant following the forward ant senses the pheromone and follows it.

4. In this way ant behind the first ant gets to know the route follows by the ant and follows it.

5. All the ants follow the same procedure and travel in a straight line.

6. In this way all the ants reach their destination using the shortest routing path.

\section{RESULT AND ANALYSIS}

Leach protocol

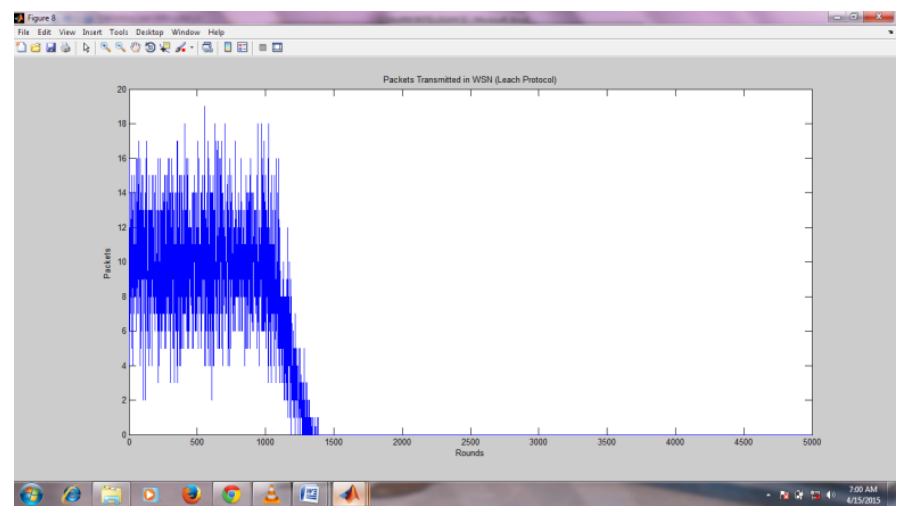

Figure 7 - Packets transmitted in leach protocol

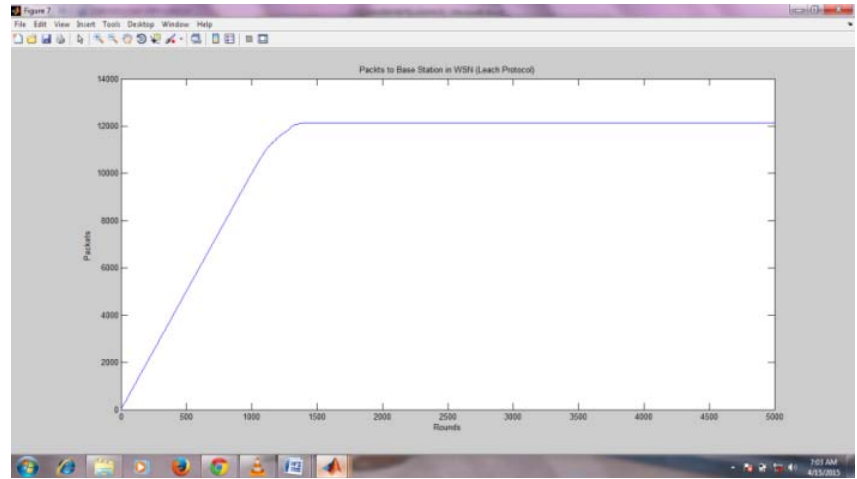

Figure 8 - Packets transmitted to BS in leach protocol

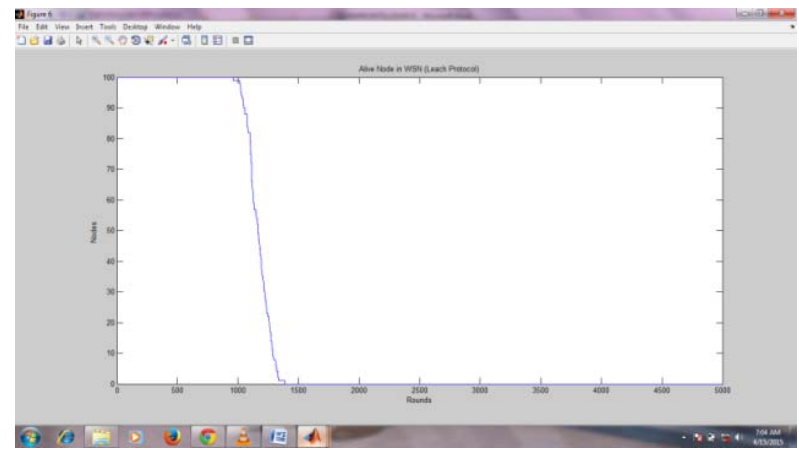

Figure 9 - Alive nodes in leach protocol

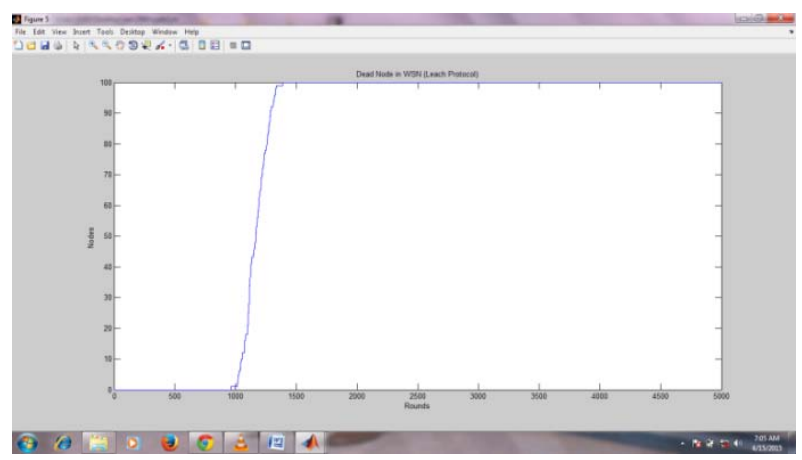

Figure 10 - Dead nodes in leach protocol

Pso algorithm

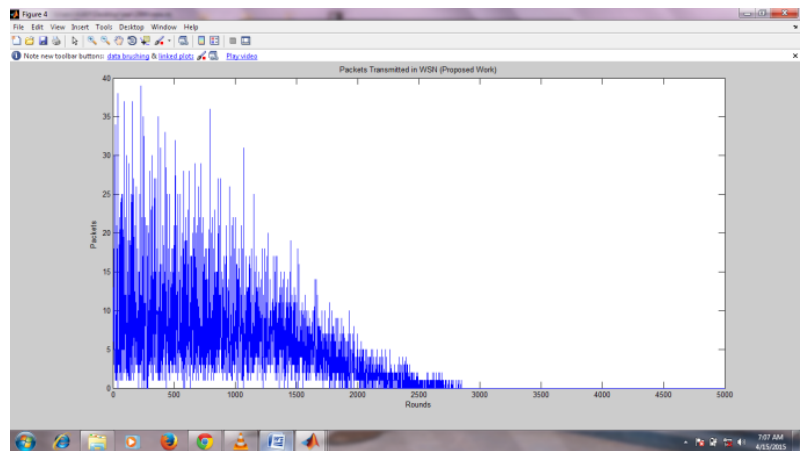

Figure 11 - Packets transmitted in proposed work 

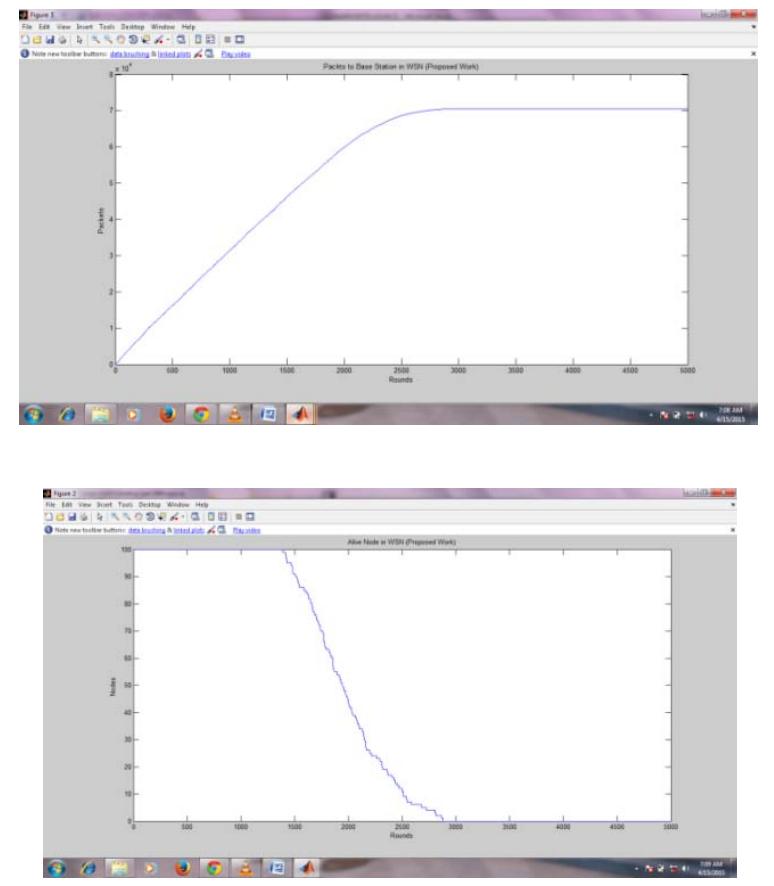

Figure 13 - Alive nodes in proposed work

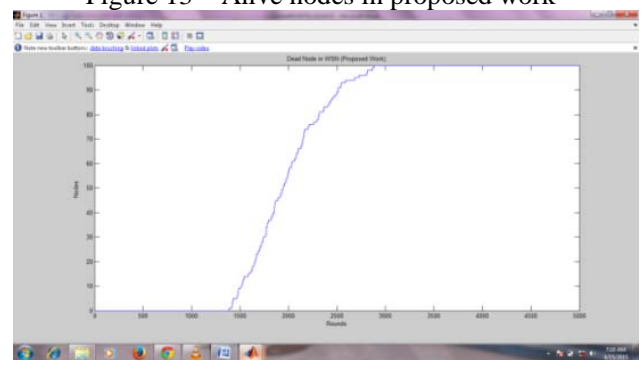

Figure 14 - Dead nodes in proposed work.

A efficient ant bee colonybased routing algorithm for Mobile Ad hoc Networks is obtained. Shortcomings in implementation of above algorithm are also discussed. Also the relatively novel domain of ant bee colonyoffers algorithmic design principles, inspired by complex adaptive biological systems, that well match the constraints and the challenges of WSNs. Therefore, a number of routing protocols for WSNs have been developed in the last years based on SI principles, and, more specifically, taking inspiration from foraging behaviors of ant and bee colonies. In this paper, we have presented a rather extensive survey of these SI-based algorithms for routing in WSNs. Wireless sensor networks consist of large sets of resource-constrained nodes. The design of effective, robust, and scalable routing protocols in these networks is a challenging task. On the other hand, the relatively novel domain of ant bee colony offers algorithmic design principles, inspired by complex adaptive biological systems, that well match the constraintsand the challenges of WSNs. Therefore, a number of routing protocols for WSNs have been developed in the last years based on SI principles, and, more specifically, taking inspiration from foraging behaviors of ant and bee colonies. In this paper, we have presented a rather extensive survey of these SI-based algorithms for routing in WSNs. We have also pointed out a number of methodological flaws in the way these algorithms are commonly presented and empirically evaluated

\section{REFERENCES}

1. A. Kaur, and S. Goyal, "A Survey on the Applications of Bee Colony Optimization Techniques”, International Journal On Computer Science And Engineering, Vol. 3, No. 8 , pp.30373045 Aug-2011.

2. A. Kaur, and S. Goyal, "The Applications Survey : Bee Colony”, Engineering Science and Technology: An International Journal (ESTIJ), Vol.2, No. 2, April 2012.

3. L. P. Wong, M. Y. H. Low and C. S. Chong, "Bee Colony Optimization with Local Search for Travelling Salesman Problem,” International Journal on Artificial Intelligence Tools (IJAIT), vol. 19, pp. 305-334, 2010.

4. Hawbani et. Al.,"Wireless Sensor Network Routing Based on Sensors Grouping”, Wireless Sensor Network,2014, 6, 8-17

5. H. L. Nguyen and U. T. Nguyen, "Study of Different Types of Attacks on Multicast in Mobile Ad Hoc Net- works," Networking, International Conference on Sys- tems and International Conference on Mobile Communi- cations and Learning Technologies, 23-29 April 2006, 149 p.

6. Kumar et. Al.,"Simulation \& Performance Evaluation of QoS Routing Protocol for Ad-hoc Networks Using Directional Communication”, Int. J. Communications, Network and System Sciences,2012, 5, 825-833

7. Meng et. Al.,"Swarm Intelligence in Power System Planning”, International Journal of Clean Coal and Energy, 2013, 2, 1-7

8. R. H. Jhaveri, S. J. Patel, et al., "DoS Attacks in Mobile Ad Hoc Networks: A Survey,” 2nd International Con- ference on Advanced Computing \& Communication Tech- nologies, 2012.

9. S. Dabideen, B. R. Smith and J. J. Garcia-Luna-Aceves, “An End-to-End Solution for Secure and Survivable Rout- ing in MANETs," 7th International Workshop on Design of Reliable Communication Networks, Washington DC, 25-28 October 2009, PP. 183-190.

10. V. Gokhale, S. K. Ghosh, et al., "Classification of At- tacks on Wireless Mobile Ad Hoc Networks and Vehicu- lar Ad Hoc Networks," Security of Self-Organizing Net- works, Auerbach Publications: MANET, WSN, WMN, VANET, 2010, p. 195.

11. Guikai Liu et. Al., Subarea Tree Routing (STR) in Multi-hop Wireless Ad hoc Networks, Wireless Sensor Network, 2009, $1,1-60$

12. Minghui Li et. Al., Accurate Angle-of-Arrival Measurement Using Particle Swarm Optimization Wireless Sensor Network, 2010, 2, 358-364

Sathya P. Duraisamy et. Al., A New Multilevel Thresholding Method Using Swarm Intelligence Algorithm for Image Segmentation, J. Intelligent Learning Systems \& Applications, 2010, 2, 126-138

13. Ka Lun Lam et. Al., A Study of Address Shortage in a Tree Based ZigBee Network for Mobile Health Applications, Wireless Sensor Network, 2012, 4, 147-153

14. Wei Huang et. Al., Projection Pursuit Flood Disaster Classification Assessment Method Based on Multi-Swarm Cooperative Particle Swarm Optimization, Journal of Water Resource and Protection, 2011, 3, 415-420

15. Mark Hartonget. Al., Secure Interchange Routing, Journal of Transportation Technologies, 2011, 1, 21-29

16. Zhizhong Jieet. Al., Opportunistic Unicast and Multicast Routing Protocol for VANET, Journal of Software Engineering and Applications, 2013, 6, 319-327

17. Fatemeh Ayatollahiet. Al., A new hybrid particle swarm optimization for mutlimodal brain image registration , J. Biomedical Science and Engineering, 2012, 5, 153-161

18. Olatunde Abiona et. Al. ,Wireless Network Security: The Mobile Agent Approach, Int. J. Communications, Network and System Sciences, 2013, 6, 443-450 
19. Majing Su et. Al. , A Measurement Study on Resource Popularity and Swarm Evolution of BitTorrent System, Int. J. Communications, Network and System Sciences, 2013, 6, 300-308

20. Wenping Chang et. Al., A Novel Particle Swarm Optimization for Optimal Scheduling of Hydrothermal System Energy and Power Engineering, 2010, 2, 223-229

21. Magdi S. Mahmoud et. Al. , Design of Robust Power System Stabilizer Based on Particle Swarm Optimization , Circuits and Systems, 2012, 3, 82-89
22. Chen et. Al , Artificial Searching Swarm Algorithm and Its Performance Analysis, Applied Mathematics, 2012, 3, 14351441

23. Siddarth et. Al, A Cluster Based QoS-Aware Service Discovery Architecture Using Swarm Intelligence, Communications and Network, 2013, 5, 161-168

24. Sangeetha et. Al., QoS Aware Routing Protocol to Improve Packet Transmission in Shadow-Fading Environment for Mobile Ad Hoc Networks, Communications and Network, 2013, 5, 611-617 